\title{
Klinik und operative Therapie des zervikalen Syndroms
}

\author{
Eckhard Rickels, Kathrin König, Martin Lorenz, Hans-Jörg Oestern
}

\section{Zusammenfassung}

Degenerative Veränderungen der Halswirbelsäule, die die Nervenabgänge (Wurzeln) bedrängen, führen zum Beschwerdebild des zervikalen Wirbelsäulensyndroms. Wird der Spinalkanal als Ganzes eingeengt, führt dies zur zervikalen Spinalkanalstenose. Ursächlich sind Bandscheibenvorfälle, knöcherne Einengungen der Wurzelabgänge oder knöcherne oder ligamentäre Anbauten im Spinalkanal. Die Diagnose und Höhenlokalisation kann durch die radikuläre Symptomatik in der Regel eindeutig gestellt werden. Mittel der Wahl bei der Bildgebung ist die Magnetresonanztomografie (MRT). Der dorsale und der heute favorisierte ventrale Zugang zum Spinalkanal ermöglichen das sichere Abtragen von Bandscheibenvorfällen, der knöchernen Anbauten oder Einengungen der Wurzelabgänge. Längere knöcherne Stenosen der HWS erfordern ggf. den Wirbelkörperersatz.

\section{Clinical and Surgical Therapy for Cervical Spine Syndrome}

Degenerative alterations of cervical nerve roots are the cause of cervical spine syndrome. Alterations due to disc herniation, osteophytic spurs or ligament hypertrophy result in stenosis of the spinal canal. The diagnosis and level detection can easily be deduced from the radiculopathy with pain, hypaesthesia and motor weakness of the specific nerve roots. MRI is the first choice in imaging diagnostics. For surgery, the dorsal as well as the nowadays favoured ventral approaches allow a safe removal of the disc as well as of the osteophytic spurs. Long-range cervical stenosis often requires a replacement of the afflicted vertebral body.

\section{Einleitung}

Die degenerativen Erkrankungen der HWS beinhalten zum einen die zervikalen Bandscheibenvorfälle, den sog. „weichen Vorfall“, zum anderen das Bild der zervikalen Stenose. Ursache hierfür kann eine kongenitale Stenose der HWS sein. Wesentlich häufiger sind dies degenerative Veränderungen mit einer Mischung aus osteophytären Anbauten, Bandscheibenvorfall und Hypertrophie der Wirbelbögen sowie der Ligamente und Facettengelenke.

OP-JOURNAL 2009; 25: 82-86

(c) Georg Thieme Verlag KG Stuttgart · New York DOI 10.1055/s-0029-1186076

\section{Klinische Symptome}

Die Schmerzstraße in das Dermatom und die Schwäche im Kennmuskel sowie die Abschwächung des entsprechenden Reflexes ermöglichen es, die geschädigte Wurzel zu identifizieren.

Fast alle degenerativen Veränderungen der Halswirbelsäule (HWS) führen zu einer schmerzhaften Bewegungseinschränkung der Halswirbelsäule mit einer Steilstellung, einem paravertebralen muskulären Hartspann, einem lokalen Klopf- oder Druckschmerz über der Wirbelsäule, Schmerzen beim Überstrecken der HWS, weniger bei Beugung.

Der Schmerz wird als Nackenschmerz beschrieben, meist auch als Nackenkopfschmerz und/oder als Schmerzausstrah- lung in den Arm. Kopfbewegungen verstärken meist die Beschwerden. Die Patienten versuchen durch eine Schonhaltung, oft Streckstellung der HWS, Linderung zu erlangen. Das Auflegen des Armes auf den Kopf bringt oft auch eine Schmerzlinderung.

Zusätzlich geben die Patienten ein Schweregefühl des Armes an.

Ein Bandscheibenvorfall führt normalerweise zu einer Bedrängung einer Nervenwurzel auf dem Niveau der Herniation (z. B. führt ein Vorfall C6/C7 zu einer Symptomatik der Nervenwurzel C7). Stärkere Beschwerden führen deshalb zur Schmerzausstrahlung entsprechend dem Versorgungsgebiet eines Nervs (Dermatom). Entsprechend dem Versorgungsgebiet des Nervs kommt es zu einer Abschwächung oder zu einem Ausfall der zugehörigen Kennreflexe und zu Lähmungen.

Es ist also anhand der Schmerzstraße in das Dermatom und der Schwäche im Kennmuskel sowie der Abschwächung des entsprechenden Reflexes in der Regel möglich, die geschädigte Wurzel zu identifizieren (Abb. 1).

Die beschriebenen Beschwerden werden durch bedrängende Einengung des Spinalkanals oder der Nerven bei Austritt aus dem Spinalkanal (Foramina) verursacht. Diese Bedrängung der Nervenstrukturen kann durch Bandscheibenmaterial, durch eine Bandhypertrophie oder durch knöcherne Anbauten (Osteophyten) bedingt sein.

Hiervon abzugrenzen ist die zervikale Spinalkanalstenose.

Die Zervikalstenose schädigt nicht nur eine Wurzel, sondern durch die in den Spinalkanal schauenden knöchernen Anbauten, die Hypertrophie der Bänder oder den Bandscheibenvorfall, das ganze Rückenmark (Myelopathie). Hier kann 


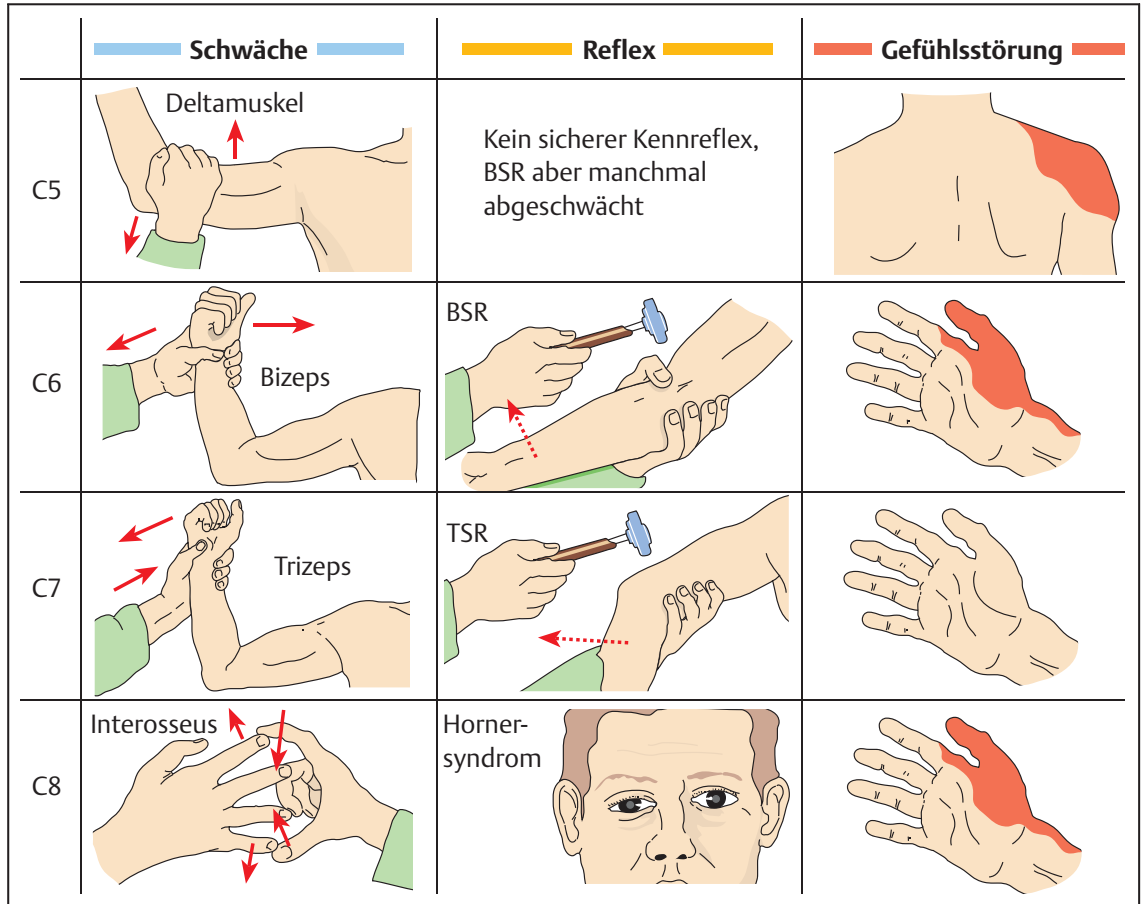

Abb. 1 Schmerzstraße und Reflexdifferenz sowie Schwäche des Kennmuskels erlauben die Identifizierung der bedrängten Wurzel.

es, je nach Läsionshöhe, zu Störungen der Feinmotorik, bei den Beinen zu einer Gangunsicherheit kommen. Die sich langsam verschlechternden Lähmungen der Arme/Beine können auch zu einer Tetraparese führen. Auch Blasenfunktionsstörungen können auftreten.

Einige Patienten haben ein LhermitteZeichen (elektrisierende schockartige Empfindungen entlang der Wirbelsäule), das als Zeichen einer Rückenmarksschädigung gewertet wird. Der Verlauf dieser Schädigung ist nicht absehbar, er kann rasch progredient oder schubweise erfolgen [8].

Führen Veränderungen der HWS zu einer Rückenmarksschädigung, ist der Verlauf dieser Schädigung nicht absehbar; er kann rasch progredient oder schubweise erfolgen.

\section{Differenzialdiagnose}

Der akut einsetzende Nackenkopfschmerz kann als Zeichen der Subarachnoidalblutung fehlgedeutet werden. Die Qualität des Schmerzes wird hier in der Regel aber als wesentlich bedrohlicher, dramatischer als beim HWS-Syndrom angegeben. Ein CT des Kopfes bringt Klarheit.
Tumoren des Halsmarks, der Wirbelsäule oder der umgebenden Weichteile können durch Komprimierung der Nerven die oben beschriebenen Beschwerden hervorrufen, ebenso Entzündungsprozesse. Weitere differenzialdiagnostische Erwägungen betreffen das kostoklavikuläre Syndrom, Karpaltunnelsyndrom, Ulnarisrinnensyndrom, Periarthritis humeroscapularis oder einen Pancoast-Tumor. Auch hier bringen die entsprechende Bildgebung oder die Neurophysiologie eine Klärung.

\section{Bildgebung}

Mittel der 1. Wahl bei der Bildgebung von Schädigungen im zervikalen Spinalkanal ist die Magnetresonanztomografie.

Mittel der 1 . Wahl bei der Bildgebung ist die Magnetresonanztomografie (MRT) als nicht invasive Diagnostik, mit guter Darstellung der weichen (nicht verkalkten) Bandscheibenvorfälle, Bandhypertrophien, Verlagerungen des Rückenmarks und der Myelopathie (Abb. 2).

Wenn kein MRT durchgeführt werden kann (z.B. Platzangst oder Herzschrittmacher), ist die normale Computertomografie oft zur Diagnosestellung ausreichend, insbesondere knöcherne Ein-

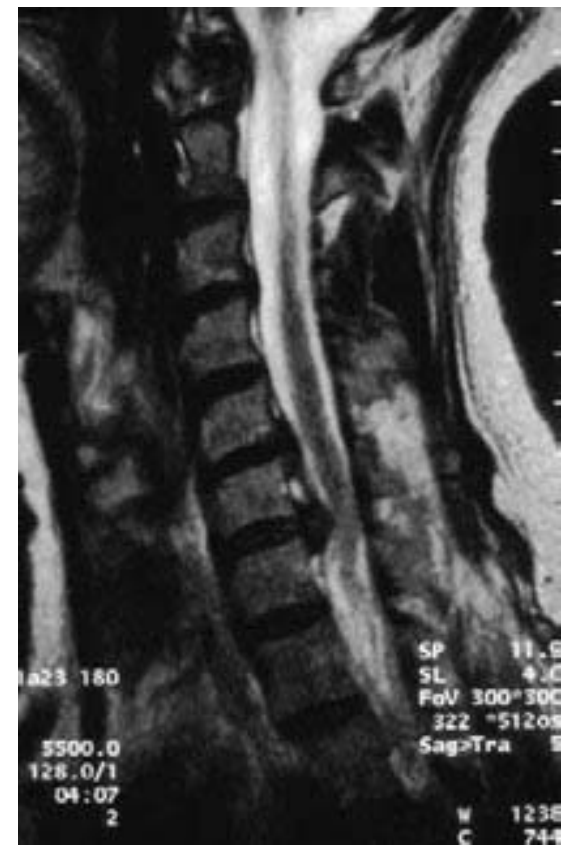

Abb. 2 Bandscheibenvorfall mit Verdrängung des Rückenmarks im MRT.

engungen werden oft deutlicher dargestellt als im MRT.

In den wenigen Fällen, bei denen mit MRT und/oder CT keine eindeutige Diagnostik durchgeführt werden kann, ist die zervikale Myelografie zu erwägen (Abb. 3).

Ein wasserlösliches Kontrastmittel wird intrathekal (in der Regel über eine Lumbalpunktion) appliziert und anschließend werden normale Röntgenaufnahmen in verschiedenen Ebenen durchgeführt, ebenso wie ein CT. Dieses Myelo-CT stellt die Wurzelabgänge eindeutig dar. Eine Bedrängung des Wurzelabgangs wird als Fehlen der Wurzel abgebildet.

Bei der Bildgebung ist zu beachten, dass die Mehrzahl aller Patienten über 50 Jahre radiologisch nachweisbare eindeutige degenerative Veränderungen der HWS hat. Nur ein kleiner Teil der Patienten hat jedoch operationswürdige Beschwerden [5].

\section{Konservative Behandlung}

90\% (!) aller Patienten mit dem klinischen Bild einer zervikalen Wurzelschädigung (Radikulopathie) zeigen eine Erholung ohne chirurgischen Eingriff [9]. Konservative Behandlungsmaßnahmen 


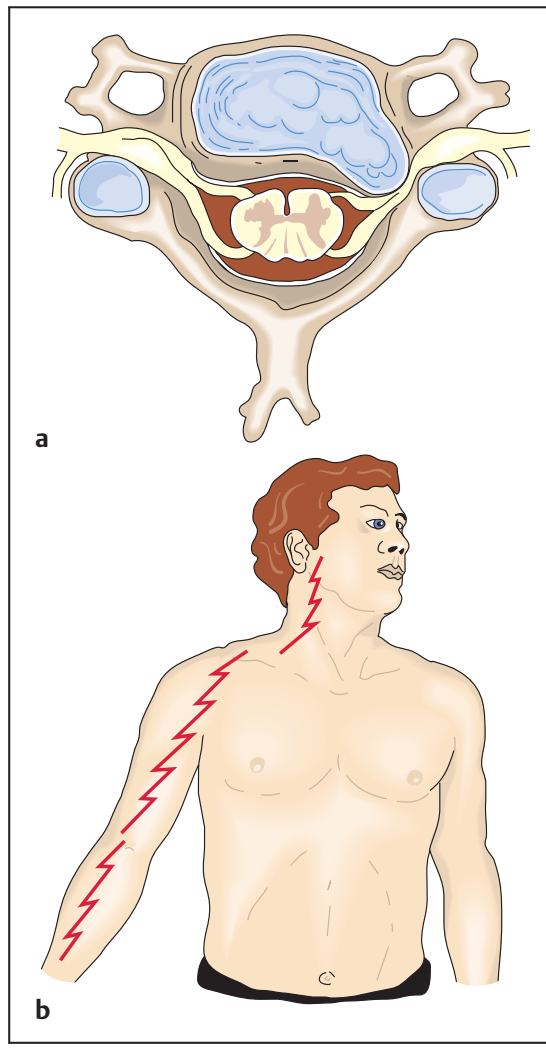

Abb. $\mathbf{3}$ a und $\mathbf{b}$ Zervikaler Bandscheibenvorfall (a) mit entsprechender Klinik (b).

mit ausreichender Schmerzmedikation, vorsichtiger Krankengymnastik und Bewegungsschulung sind also Erfolg versprechend.

\section{Operationsindikation}

Eine Operationsindikation stellen die Zeichen der Myelopathie, klinisch oder im MRT, dar, da eine langfristige Schädigung des Rückenmarks zu irreparablen Schäden bis zum Querschnittsyndrom führen wird. Nicht zu beherrschende Schmerzen sind eine Operationsindikation sowie eine neurologische Verschlechterung. Aber auch immer wiederkehrende Schmerzattacken können eine OP-Indikation darstellen.

\section{Operationtechniken}

Das Abtragen der den Spinalkanal und/ oder die Wurzel einengenden Osteophyten oder des Bandscheibenmaterials kann prinzipiell auf 2 Wegen geschehen.

Eine Methode stellt die Operation nach Frykholm dar. Hier wird über einen dorsalen Zugang zur Halswirbelsäule eine (Teil-)Hemilaminektomie durchgeführt und von dorsal der in der Regel ventral sitzende Prolaps entfernt oder eine Fora-

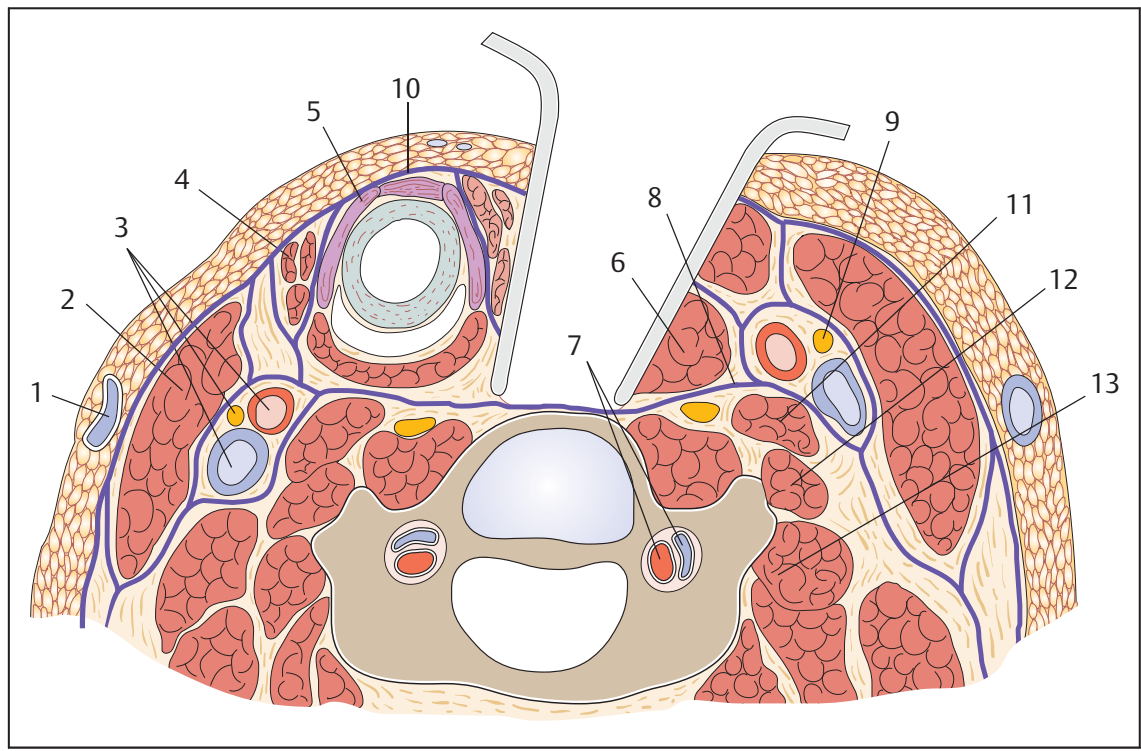

Abb. 4 Zugangswege zur HWS (nach Mohsenipour, Goldhahn, Fischer, Plartzer, Pomaroli: Operative Zugangswege in der Neurochirurgie, Thieme 1997, S. 131, Abb 172).

minotomie durchgeführt, die der Wurzel Platz schafft.

Vorteil der Operation ist der einfache $\mathrm{Zu}-$ gang. Nachteil der Technik ist, dass die Mehrzahl der Patienten nicht nur einen freien sequestrierten Bandscheibenvorfall hat, sondern Veränderungen, bestehend aus Osteophyten und Bandscheibenmaterial sowie Bandverdickungen. Diese Anbauten und Verdickungen liegen aber vor der Ventralseite des Rückenmarks. Im Gegensatz zum Lumbalbereich ist es aber nicht möglich, den Duralschlauch zur Seite zu drängen. Deshalb hat sich der ventrale Zugang zur Halswirbelsäule in verschiedenen Variationen $[3,10]$ durchgesetzt.

Beim liegenden Patienten wird bei überstrecktem Kopf rechts (gering erhöhtes Risiko einer Verletzung des N. recurrens mit Heiserkeit) oder links ein Hautschnitt gesetzt, das Unterhautgewebe mobilisiert, das Platysma gespalten und der Rand des M. sternocleidomastoideus dargestellt.

Im Wesentlichen stumpf kann man dann, das Gefäß-Nerven-Paket mit A. carotis, V. jugularis und $N$. vagus nach lateral haltend, direkt zur Halswirbelsäule gelangen.

Trachea und Ösophagus müssen geschont werden.

Dann wird der M. longus zervicis dargestellt und $\mathrm{zu}$ den Seiten weggeschoben. Es erfolgt z.B. durch Punktion und
Röntgenbild Sicherung der zu operierenden Höhe (Abb. 4).

Durch Einbringen des Caspar-Sperrers mit den Schrauben in den darüber- und darunterliegenden Wirbeln kann das inzidierte Bandscheibenfach etwas aufgespreizt werden. Ein Valvensperrer erleichtert den Überblick.

Anschließend wird das Bandscheibenfach ausgeräumt.

Die Lichtverhältnisse und der Überblick bei Benutzung eines Operationsmikroskops erleichtern das weitere Vorgehen. Nach dem Ausräumen des Bandscheibenfachs und dem Anfrischen der Deckplatten gewinnt man Zugang zum hinteren Längsband. Sollte das Fach zu klein sein, um Überblick zu erlangen, kann mit der Fräse der Zugang erweitert werden. Man erkennt die osteophytären Anbauten, Lücken im Längsband und eventuell einen freien Sequester. Jetzt lässt sich der Vorfall herausziehen. Gegebenenfalls erfolgt durch Stanzen oder Fräsen eine Erweiterung. Dann erfolgt das Abtragen der Osteophyten und Weichteilverdickungen. Die Wurzelabgänge werden identifiziert und nach lateral verfolgt, die Foramina werden, wenn nötig, erweitert. Liegen Duralsack und Wurzeln frei, erfolgt der Verschluss des Bandscheibenfachs.

Dies kann mit einem Knochendübel (Beckenkamm), mit Palacosplombe oder Cages aus PEEK, aus Titan oder ähnlichem Material geschehen, der der Fusion 


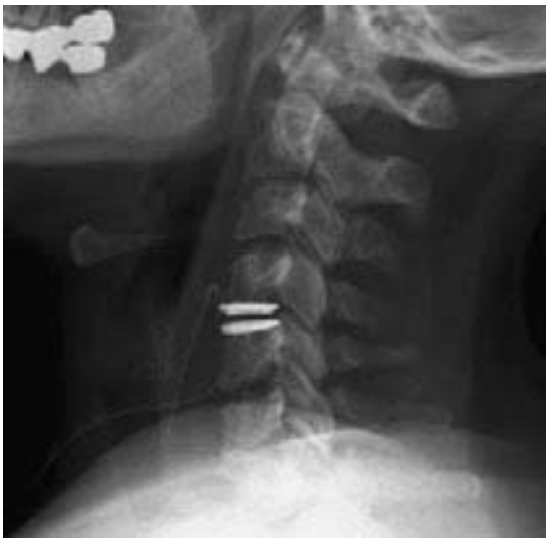

Abb. 5 Eingebrachte Bandscheibenprothese.

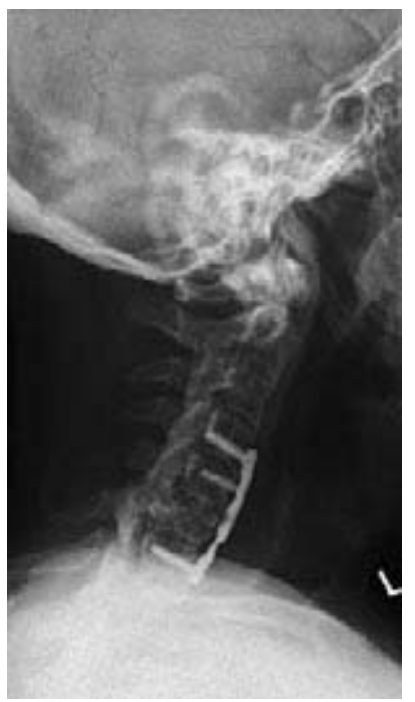

Abb. 6 Intraoperatives Bild: Fusionierung des Zwischenwirbelraums mit einem Beckenkammdübel und Verplattung.

der beiden angrenzenden Wirbelkörper dient. Derzeit müssen alle Techniken als gleichwertig angesehen werden [1].

Ob die Bandscheibenprothese hier langfristig bessere Ergebnisse zeigt, insbesondere durch Schonung der angrenzenden Bandscheibenfächer vor Überlastung, ist noch unklar (Abb. 5).

Es besteht auch noch eine Diskussion, ob die Verplattung zur Sicherung der Fusion notwendig ist [4] (Abb. 6).

Nach traumatischen Bandscheibenvorfällen, nach intraspinalen Hämatomen oder Zerreißungen der Bänder wird immer eine Sicherung durch eine Verplattung durchgeführt. Betrifft die spinale Enge mehrere Bandscheibenetagen, kann dies in einer Sitzung operiert werden. Bei solchen multisegmentalen Ein-

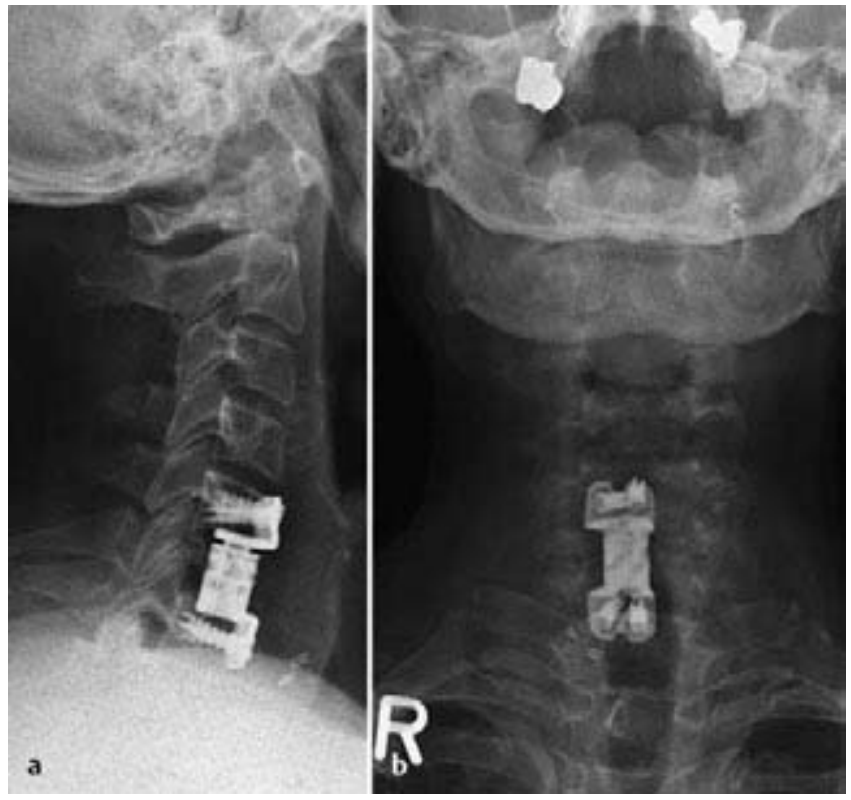

Abb. 7 a und $b$

Wirbelkörperersatz nach weiträumiger Spinalkanalstenose mit einem Titankorb und Verschraubung. engungen muss oft so viel von den Wirbelkörpern weggenommen werden, dass es sinnvoll erscheint, die gesamten Wirbelköper zu entfernen und durch Implantate zu ersetzen (Abb. 7).

Anschließend erfolgt die Einlage einer Drainage, Platysmanaht und Subkutanund Hautnaht sowie Verband. Die Anlage einer Halskrawatte ist üblich.

\section{Komplikationen}

Nachblutungen oder Schwellung nach heftiger Retraktion können zur Einengung des Atemwegs führen und damit akut lebensbedrohlich sein. Schluckstörungen sind kurz nach der OP häufig, sollten dann aber schnell abnehmen.

Schluckstörungen mit Abhusten von Nahrung weisen auf Verletzungen von Pharynx, Trachea und Ösophagus hin.

Heiserkeit ist verdächtig auf eine Schädigung des N. recurrens (11\% temporär, 4\% permanent) [7].

Intraoperativ kann die A. vertebralis verletzt werden. Dies führt zu heftigen, schwer zu stillenden Blutungen, kann zur Bildung von Pseudoaneurysmen oder Nachblutungen führen, die dann auch einen Druck auf das Rückenmark ausüben können. Thrombembolien in das Gehirn können von solchen Verletzungen ausgehen. Gleiches gilt für Verletzungen der A. carotis.
Verletzungen der Dura führen zu einem Liquorleck, das oft schwierig zu versorgen ist.

Direkte Verletzungen des Rückenmarks durch die Operation können im schlimmsten Fall zu einer Querschnittsymptomatik führen.

Eine postoperative neu auftretende Schwäche in den Armen oder Beinen deutet auf eine Verletzung von Rückenmark oder Nerven hin.

Postoperativ kann es zu einem Herausdrücken des Dübels meist durch Überstreckung des Halses kommen (Vorsicht: Physiotherapie).

Viele Patienten klagen für eine begrenzte Zeit über eine Missempfindung in den Schultern.

Nach der Wundheilung und vorsichtiger Krankengymnastik ohne Bewegungen des Kopfes ist die schnelle Wiedereingliederung problemlos.

\section{Ergebnissse}

Verschiedene Studien haben ein exzellentes Ergebnis nach Bandscheibenoperationen $[2,6]$ gezeigt. Bei der zervikalen Stenose mit Myelopathie ist das primäre Ziel, die weitere neurologische Verschlechterung zu stoppen. Ob eine neurologische Verbesserung möglich ist, ist präoperativ nicht absehbar [8]. 


\section{Literatur}

${ }^{1}$ Bärlocher CB, Barth A, Krauss JK et al. Comparative evaluation of microdiscectomy only, autograft fusion, polymethylmethacrylate interposition, and threaded titanium cage fusion for treatment of single-level cervical disc disease: a prospective randomized study in 125 patients. Neurosurg Focus 2002; 12: 1-7

2 Bohlmann H, Emery SE, Goodfellow DB et al. Robinson anterior cervical discectomy and arthrodesis for cervical radiculopathy. J Bone Joint Surg [Am] 1993; 75: 1298-1307

3 Cloward RB. The anterior approach for removal of ruptured cervical disks. J Neurosurg 1958; 15: 602-614

${ }^{4}$ Connolly PJ, Esses SI, Kostuik JP. Anterior cervical fusion: outcome analysis of patients fused with and without anterior cervical plates. J Spinal Disord 1996; 3: 202-206

5 Cusick JF. Pathophysiology and treatment of cervical spondylottic myelopathy. Clin Neurosurg 1989; 37: 661-681
${ }^{6}$ Gregorius FK, Estrin T, Crandall PH. Cervical spondylotic radiculopathy and myelopathy: a long-term follow-up study. Arch Neurol 1976; 33: 618-625

${ }^{7}$ Netterville JL, Koriwchak MJ, Winkle M. Vocal fold paralysis following the anterior approach to the cervical spine. Ann Oto Rhinol Laryngol 1996; 105: 85-91

${ }^{8}$ Nurick $S$. The natural history and the results of surgical treatment of the spinal cord disorder with cervical spondylosis. Brain 1972; 95: 101-108

${ }^{9}$ Saal J, Saal Y, Yurth E. Nonoperative management of heriated cervical intervertebral disc with radiculopathy. Spine 1996; 21: 18771883

${ }^{10}$ Smith GW, Robinson RA. The treatment of certain cervical spine disorders by anterior removal of the intervertebral disc and interbody fusion. J Bone Joint Surg [Am] 1958; 40: $607-623$
Prof. Dr. med. Eckhard Rickels

Leitender Arzt

Dr. med. Kathrin König

Lt. Oberärztin

Prof. Dr. med. Martin Lorenz

Oberarzt

Prof. Dr. med. Hans-Jörg Oestern

Chefarzt Unfallchirurgie

Bereich Neurochirurgie

Klinik für Unfallchirurgie,

Orthopädie und Neurotraumatologie des AKH Celle

Siemensplatz 4

29223 Celle

eckhard.rickels@akh-celle.de 